\title{
A Characterization of Polynomial Density on Curves via Matrix Algebra
}

\author{
Carmen Escribano ${ }^{1,2, *,+}$, Raquel Gonzalo ${ }^{1, \dagger}$ and Emilio Torrano ${ }^{1, \dagger}$ \\ 1 Departamento de Matemática Aplicada a las Tecnologías de la Información y las Comunicaciones, Escuela \\ Técnica Superior de Ingenieros Informáticos, Universidad Politécnica de Madrid, Campus de Montegancedo, \\ Boadilla del Monte, 28660 Madrid, Spain; rngonzalo@fi.upm.es (R.G.); emilio@fi.upm.es (E.T.) \\ 2 Center for Computational Simulation, Universidad Politécnica de Madrid, 28660 Madrid, Spain \\ * Correspondence: cescribano@fi.upm.es \\ $\dagger$ These authors contributed equally to this work.
}

Received: 18 October 2019; Accepted: 2 December 2019; Published: 12 December 2019

check for updates

\begin{abstract}
In this work, our aim is to obtain conditions to assure polynomial approximation in Hilbert spaces $L^{2}(\mu)$, with $\mu$ a compactly supported measure in the complex plane, in terms of properties of the associated moment matrix with the measure $\mu$. To do it, in the more general context of Hermitian positive semidefinite matrices, we introduce two indexes, $\gamma(\mathbf{M})$ and $\lambda(\mathbf{M})$, associated with different optimization problems concerning theses matrices. Our main result is a characterization of density of polynomials in the case of measures supported on Jordan curves with non-empty interior using the index $\gamma$ and other specific index related to it. Moreover, we provide a new point of view of bounded point evaluations associated with a measure in terms of the index $\gamma$ that will allow us to give an alternative proof of Thomson's theorem, by using these matrix indexes. We point out that our techniques are based in matrix algebra tools in the framework of Hermitian positive definite matrices and in the computation of certain indexes related to some optimization problems for infinite matrices.
\end{abstract}

Keywords: Hermitian moment problem; orthogonal polynomials; smallest eigenvalue; measures; polynomial density

\section{Introduction}

In this paper, we consider positive Borel measures $\mu$, which are finite and compactly supported in the complex plane. We always consider non-trivial measures, that is, measures with an infinite amount of points in their support. The problem of completeness of polynomials in the Hilbert space $L^{2}(\mu)$ is the following: For a certain measure $\mu$, are polynomials dense in the space $L^{2}(\mu)$ ? In other words, denote by $P^{2}(\mu)$ the closure of the polynomials in the space $L^{2}(\mu)$; the question is under what conditions the equality $L^{2}(\mu)=P^{2}(\mu)$ is true. In the particular case of $\mu$ being the two-dimensional Lebesgue measure on an arbitrary domain $G$ and $L^{2}(G)$ the associated Hilbert space, the classical results of approximation by polynomials can be seen in, e.g., the work of Gaier [1], who explored the question of which assumptions on $G$ will be assumed to have polynomials density in $L^{2}(G)$. The related questions about the existence of approximation rational, entire, or meromorphic were solved by the great theorem of Mergelyan in 1951, which completes a long chain of theorems about approximation by polynomials.

The problem of density of polynomials is also an interest topic in the theory of orthogonal polynomials associated with a measure. Indeed, in the particular case of orthogonal polynomials in the unit circle the well-known Szegö theory (see, e.g., [2,3]) deals with the problem of polynomial approximation using proper tools of orthogonal polynomials. 
On the other hand, in [4], a necessary condition was provided to assure polynomial approximation using the behavior of the smallest eigenvalues of the finite sections of the moment matrix associated with a measure. Along this work, we follow this matrix approach in order to obtain the main results in this paper.

Throughout this paper, we consider infinite Hermitian positive definite matrices $\mathbf{M}=\left(c_{i, j}\right)_{i, j=0}^{\infty}$. As in $[4,5]$, a Hermitian positive definite matrix (HPD matrix) defines an inner product $\langle$,$\rangle in the$ space $\mathbb{P}[z]$ of all polynomials with complex coefficients in the following way: if $p(z)=\sum_{k=0}^{n} v_{k} z^{k}$ and $q(z)=\sum_{k=0}^{m} w_{k} z^{k}$, then

$$
\langle p(z), q(z)\rangle=v \mathbf{M} w^{*}
$$

being $v=\left(v_{0}, \ldots, v_{n}, 0,0, \ldots\right), w=\left(w_{0}, \ldots, w_{m}, 0,0, \ldots\right) \in c_{00}$, where $c_{00}$ is the space of all complex sequences with only finitely many non-zero entries. The associated norm is $\|p(z)\|^{2}=\langle p(z), p(z)\rangle$ for every $p(z) \in \mathbb{P}[z]$.

An interesting class of HPD matrices are those which are moment matrices with respect to a measure $\mu$, i.e., HPD matrices $\mathbf{M}=\left(c_{i, j}\right)_{i, j=0}^{\infty}$ such that there exists a measure $\mu$ with infinite support on $\mathbb{C}$ and finite moments for all $i, j \geq 0$,

$$
c_{i, j}=\int z^{i} \bar{z}^{j} d \mu
$$

Our aim here is to obtain conditions to assure polynomial approximation in Hilbert spaces $L^{2}(\mu)$, with $\mu$ a compactly supported measure in the complex plane, in terms of properties of the associated matrix M. To do it, in the more general context of Hermitian positive semidefinite matrices, we introduce two matrix indexes $\gamma(\mathbf{M})$ and $\lambda(\mathbf{M})$, each related with different optimization matrix problems. Among these indexes, we highlight the index $\gamma$ that is essential to characterize the polynomial density in our context. The other index $\lambda$ is related to the asymptotic behavior of the smallest eigenvalues in our previous works (see [4]). These indexes are introduced in Section 2 and some of their properties are given. We also provide an application to the index $\lambda$ to some problems of perturbations of measures in the same direction as in [6].

In Section 3, we consider the case when the Hermitian positive semidefinite matrices are moment matrices associated with a measure $\mu$ with compact support in the complex plane. Our main result is a characterization of completeness of polynomials in the associated space $L^{2}(\mu)$, in the case of Jordan curves with 0 in its interior, in terms of the index $\gamma$ of the moment matrix associated with the measure $\mu$.

In Section 4, we give our main result, which is the characterization of density of polynomials on Jordan curves with non-empty interior in terms of another index related to the index $\gamma$. Moreover, we provide a matrix algebra point of view of the notion of bounded point evaluation of a measure. This leads us to obtain a new proof of Thomson's theorem in $[7,8]$, in the particular context of Jordan curves with non-empty interior, using our techniques and our results.

Finally, we point out that our approach is based in matrix algebra tools in the frame of general HPD and in the computation of certain indexes related to some optimization problems for infinite matrices. This point of view would allow solving certain matrix optimization problems in terms of the theory of orthogonal polynomials and on the other hand would let obtaining results of interest concerning orthogonal polynomials using the matrix optimization tools.

\section{New Indices of an HPD Matrix and Connections with the Polynomial Approximation}

In this section, we introduce some indices associated with general Hermitian positive semidefinite matrices. Let $\mathbf{M}=\left(c_{i, j}\right)_{i, j=0}^{\infty}$ be an infinite Hermitian matrix, i.e., $c_{i, j}=\overline{c_{j, i}}$. We recall that an infinite HPD M matrix verifies that $\left|\mathbf{M}_{n}\right|>0$ for all $n \geq 0$, where $\mathbf{M}_{n}$ is the truncated matrix of size $(n+1) \times$ $(n+1)$ of $\mathbf{M}$. In an analogous way, if $\left|\mathbf{M}_{n}\right| \geq 0$ for all $n \geq 0$, we say that $\mathbf{M}$ is a Hermitian positive 
semidefinite matrix (HPSD). In the sequel, we use the same notation as in [4]; we denote by $(1, v) \equiv$ $\left(1, v_{1}, \ldots, v_{n}, 0,0, \ldots\right)$ for every $v=\left(v_{1}, v_{2}, \ldots\right) \in c_{00}$ and by $(v, 1,0, \ldots) \equiv\left(v_{0}, \ldots, v_{n-1}, 1,0, \ldots\right)$

Definition 1. Let $\mathbf{M}$ be an infinite Hermitian positive semidefinite matrix. We define

$$
\gamma(\mathbf{M})=\inf \left\{(1, v) \mathbf{M}\left(\begin{array}{c}
1 \\
v^{*}
\end{array}\right), v \in c_{00}\right\} .
$$

This index always exists and $\gamma(\mathbf{M}) \geq 0$.

Definition 2. Let $\mathbf{M}$ be an infinite Hermitian positive semidefinite matrix. We define

$$
\lambda(\mathbf{M})=\inf \left\{v \mathbf{M} v^{*} ; v v^{*}=1, v \in c_{00}\right\} .
$$

This index always exists and $\lambda(\mathbf{M}) \geq 0$.

Remark 1. Note that an important link between eigenvalue problems and optimization is the Rayleigh quotients. Indeed, for Hermitian matrices $\mathbf{M}_{n}$, it is well-known that, if we define $Q_{n}(v)=\frac{v \mathbf{M}_{n} v^{*}}{v v^{*}}$ for $0 \neq v \in \mathbb{C}^{n}$, then $\min \left\{Q_{n}(v): v \in \mathbb{C}^{n}\right\}$ and $\max \left\{Q_{n}(v): v \in \mathbb{C}^{n}\right\}$ gives the extreme eigenvalues of $\mathbf{M}_{n}$. We denote by $\lambda_{n}$ the smallest eigenvalue of $\mathbf{M}_{n}$ as in [4]; that is, if $\|\cdot\|_{2}$ is the euclidean norm in $\mathbb{C}^{n+1}$,

$$
\lambda_{n}=\inf \left\{v \mathbf{M}_{n} v^{*} ; v \in \mathbb{C}^{n+1},\|v\|_{2}=1\right\},
$$

moreover, the sequence $\left\{\lambda_{n}\right\}_{n=0}^{\infty}$ is an non-increasing sequence and

$$
\lambda(\mathbf{M})=\lim _{n \rightarrow \infty} \lambda_{n}
$$

Next, we relate these indexes.

Proposition 1. Let $\mathbf{M}$ be an infinite Hermitian semi-definite positive matrix. Then,

$$
\lambda(\mathbf{M}) \leq \gamma(\mathbf{M}) .
$$

Proof. Let $0 \neq v \in c_{00}$ and consider the normalized sequence $\frac{v}{\left(v v^{*}\right)^{1 / 2}}$. By the definition of $\lambda(\mathbf{M})$ we have that

$$
\frac{v}{\left(v v^{*}\right)^{1 / 2}} \mathbf{M} \frac{v^{*}}{\left(v v^{*}\right)^{1 / 2}} \geq \lambda(\mathbf{M})
$$

By taking in mind that, for any $t>0,(t v) \mathbf{M}(t v)^{*}=t^{2} v \mathbf{M} v^{*}$ holds, then it easily follows that

$$
v \mathbf{M} v^{*} \geq \lambda(\mathbf{M}) v v^{*}
$$

Consequently,

$$
(1, v) \mathbf{M}\left(\begin{array}{c}
1 \\
v^{*}
\end{array}\right) \geq \lambda(\mathbf{M})(1, v)(1, v)^{*} \geq \lambda(\mathbf{M}) .
$$

By taking the infimum, we obtain $\lambda(\mathbf{M}) \leq \gamma(\mathbf{M})$.

Remark 2. The equality $\lambda(\mathbf{M})=\gamma(\mathbf{M})$ is not true in general, even for Toeplitz matrices. For instance, consider the matrix 


$$
\mathbf{T}=\left(\begin{array}{cccccc}
1 & -1 / 2 & 1 / 4 & -1 / 8 & 1 / 16 & \ldots \\
-1 / 2 & 1 & -1 / 2 & 1 / 4 & -1 / 8 & \ldots \\
1 / 4 & -1 / 2 & 1 & -1 / 2 & 1 / 4 & \ldots \\
-1 / 8 & 1 / 4 & -1 / 2 & 1 & -1 / 2 & \ldots \\
1 / 16 & -1 / 8 & 1 / 4 & -1 / 2 & 1 & \ldots \\
\vdots & \vdots & \vdots & \vdots & \vdots & \ddots
\end{array}\right)
$$

By induction, we obtain that $\left|\mathbf{T}_{n}\right| /\left|\mathbf{T}_{n-1}\right|=3^{n} / 4^{n}$; consequently, $\kappa^{2}=\lim _{n}\left|\mathbf{T}_{n-1}\right| /\left|\mathbf{T}_{n}\right|=4 / 3$, where $\kappa_{n}$ is the leading coefficient of orthonormal polynomial sequence and $\kappa=\lim _{n} \kappa_{n}$. We obtain $\rho(t)$, which is the absolutely continuous part of the associated measure of the Toeplitz matrix $\mathbf{T}$,

$$
\rho(t)=\sum_{n=-\infty}^{\infty} \frac{(-1)^{n}}{2^{|n|}} e^{i n t}=\frac{3 e^{i t}}{\left(2+e^{i t}\right)\left(2 e^{i t}+1\right)}=\frac{3}{5+4 \cos (t)} .
$$

It follows from [9] that

$$
\lambda(\mathbf{T})=\min _{t \in[0,2 \pi]} \rho(t)=\frac{1}{3} .
$$

Therefore, according to [4], it can be obtained that

$$
\gamma(\mathbf{T})=\frac{1}{\kappa^{2}}=\frac{3}{4}
$$

Note that this example shows how to use our techniques to solve certain matrix optimization problem.

In the set of infinite Hermitian positive semidefinite matrices, we may define an order in the following way: we say that $\mathbf{M}_{1} \leq \mathbf{M}_{2}$ if $v \mathbf{M}_{1} v^{*} \leq v \mathbf{M}_{2} v^{*}$ for every $v \in c_{00}$. The following results are directly obtained from the definition.

Lemma 1. Let $\mathbf{M}_{1}, \mathbf{M}_{2}$ be infinite Hermitian positive semidefinite matrices with $\mathbf{M}_{1} \leq \mathbf{M}_{2}$; then:

1. $\lambda\left(\mathbf{M}_{1}\right) \leq \lambda\left(\mathbf{M}_{2}\right)$.

2. $\gamma\left(\mathbf{M}_{1}\right) \leq \gamma\left(\mathbf{M}_{2}\right)$.

We give some applications of the above result to some perturbation results in the same lines as [6]. Let $\sigma$ be a non-trivial positive measure with support in $\mathbb{T}=\{z \in \mathbb{C}:|z|=1\}$, which verifies Szegö condition, that is $\int_{0}^{2 \pi} \ln (\rho(\theta)) d \theta>-\infty$, where $\rho(\theta)$ is the absolutely continuous part of $\sigma$ in the Lebesgue decomposition (see [10]). In [6], it is obtained that, if the measure $\sigma$ verifies Szegö condition and $\widetilde{\sigma}$ is the perturbed measure of $\sigma$ by the normalized Lebesgue measure in the unit circle, that is $d \widetilde{\sigma}=d \sigma+r \frac{d \theta}{2 \pi}$ for $r>0$, then $\widetilde{\sigma}$ also verifies Szegö condition. Using our techniques, we generalize this result pointing out that there is no need to require that $\sigma$ verifies Szegö condition since the conclusion is true always. Indeed we have the next result.

Corollary 1. Let $\sigma_{1}, \sigma_{2}$ positive measures with support on $\mathbb{T}$ Assume that one of them verifies Szegö condition, then the measure $\sigma:=\sigma_{1}+\sigma_{2}$ verifies Szegö condition. In particular, if $d \widetilde{\sigma}=d \sigma+r \frac{d \theta}{2 \pi}$ with $r>0$ for some positive measure with support in $\mathbb{T}$, then $\widetilde{\sigma}$ verifies Szegö condition.

Proof. Let $\mathbf{T}_{1}, \mathbf{T}_{2}, \mathbf{T}_{\sigma}$ be the Toeplitz positive semidefinite moment matrices associated with $\sigma_{1}, \sigma_{2}, \sigma$. Assume that $\sigma_{1}$ verifies Szegö condition, then $\gamma\left(\mathbf{T}_{1}\right)>0$. By Lemma 1, it follows that $\gamma\left(\mathbf{T}_{\sigma}\right) \geq$ $\gamma\left(\mathbf{T}_{1}\right)>0$ and consequently $\sigma$ verifies Szegö condition. 
Remark 3. Note that, in Corollary 1, it is not required that both measures are non-trivial; indeed, we may consider a perturbation by a finite amount of atomic points.

In the particular case of $d \widetilde{\sigma}=d \sigma+r \frac{d \theta}{2 \pi}$, obviously the normalized Lebesgue measure in the unit circle verifies Szegö condition and consequently $\widetilde{\sigma}$ also verifies Szegö condition independently of $\sigma$.

From now on, we consider an infinite HPD matrix $\mathbf{M}$. This matrix induces an inner product in the vector space $c_{00}$. In this way, the space $c_{00}$ endowed with such a norm is a vector space with an inner product that is not necessarily complete. The norm induced by the inner product

$$
\|v\|_{\mathbf{M}}^{2}=v \mathbf{M} v^{*}
$$

We consider the completion of this space with such norm that we denote by $P^{2}(\mathbf{M})$; we may apply Gram-Schmidt orthogonalization procedure to the canonical algebraic basis $\left\{e_{n}\right\}_{n=0}^{\infty}$ in $c_{00}$ and we obtain the unique orthonormal basis $\left\{v_{i}\right\}_{i=0}^{\infty}$ with $v_{i}=\left(v_{0, i}, \ldots, v_{i, i}, 0, \ldots\right)$ for $i \in \mathbb{N}_{0}$ and $v_{i, i}>0$. Consider $w_{n}=\frac{v_{n}}{v_{n, n}}$ the orthogonal monic vector. It is clear that $\left\|w_{n}\right\|_{\mathbf{M}}=\frac{1}{v_{n, n}}$ for every $n \in \mathbb{N}_{0}$. We denote by ${\overline{\left[e_{1}, e_{2}, \ldots\right]}}^{\mathbf{M}}$ the closed vector subspace generated by the set of vectors $e_{n}^{\prime} s$ with the norm induced by the matrix $\mathbf{M}$. We denote the distance of a vector $v$ to a subspace $E \subset P^{2}(\mathbf{M})$ as $\operatorname{dis}(v, E)=\inf \left\{\|v-w\|_{\mathbf{M}}: w \in E\right\}$.

Proposition 2. Let $\mathbf{M}$ be a Hermitian definite positive matrix. Let $\left\{e_{n}\right\}_{n=0}^{\infty}$ be the canonical basic sequence in $c_{00}$; then,

$$
\gamma(\mathbf{M})=\operatorname{dis}^{2}\left(e_{0},{\overline{\left[e_{1}, e_{2}, \ldots\right]}}^{\mathbf{M}}\right)
$$

From the results in [4], we have the following infinite dimensional version of the result in the case of Hermitian positive definite matrices.

Proposition 3. Let $\mathbf{M}$ be an HPSD matrix and let $\left\{v_{0}, v_{1}, v_{2}, \ldots\right\}$ be the orthonormal basis in $P^{2}(\mathbf{M})$ with respect the inner product induced by $\mathbf{M}$ with $v_{i}=\left(v_{0, i}, \ldots, v_{i, i}, 0, \ldots\right)$ for $i \in \mathbb{N}_{0}$ and $v_{i, i}>0$. Then,

$$
\gamma(\mathbf{M})=\frac{1}{\sum_{i=0}^{\infty}\left|v_{0, i}\right|^{2}},
$$

where the left side is zero if $\sum_{i=0}^{\infty}\left|v_{0, i}\right|^{2}=\infty$.

\section{HPSD Matrices Which Are Moment Matrices}

In this section, we consider the most important example of HPD matrices which are the moment matrices with respect to a Borel non-trivial compactly supported measure $\mu$ in the complex plane $\mathbf{M}(\mu)$. In this case, the space $c_{00}$ is replaced by the space of polynomials $\mathbb{P}[z]$ via the identification

$$
v=\left(v_{0}, \ldots, v_{n}, 0,0, \ldots\right) \equiv p(z)=v_{0}+v_{1} z+\cdots+v_{n} z^{n} .
$$

The associated norm in $\mathbb{P}[z]$ with respect to $\mathbf{M}:=\mathbf{M}(\mu)$ is the usual norm of the polynomials in the space $L^{2}(\mu)$, that is for every $p(z) \in \mathbb{P}[z]$.

$$
\|p(z)\|_{P^{2}(\mathbf{M})}^{2}=\int|p(z)|^{2} d \mu .
$$

As usual, the completion of the space of polynomials in the space $L^{2}(\mu)$ is denoted by $P^{2}(\mu)$, $\left\{\varphi_{n}(z)\right\}_{n=0}^{\infty}$ is the sequence of orthonormal polynomials, and $\left\{\Phi_{n}(z)\right\}_{n=0}^{\infty}$ is the associated sequence of monic orthogonal polynomials. We denote by $P_{0}^{2}(\mu)$ the completion of polynomials vanishing at zero. The well-known extremal properties of the monic polynomials and the $n$-kernels are just obtained by reformulating in this context Proposition 3 above, which, as pointed out, are results obtained 
by algebraic proofs in the more general context of the general Hermitian definite matrices. Indeed, rewriting the definition of $\gamma(\mathbf{M})$ in terms of polynomials, we obtain:

Lemma 2. Let $\mu$ be a measure compactly supported measure with infinite support in the complex plane and let $\mathbf{M}:=\mathbf{M}(\mu)$ be the associated moment matrix. Then,

$$
\gamma(\mathbf{M})=\operatorname{dis}\left(\mathbf{1}, P_{0}^{2}(\mu)\right),
$$

where 1 denotes $\Phi_{0}(z)$.

We need the following lemma:

Lemma 3. Let $\mu$ be a non-trivial positive compactly supported measure in $\mathbb{C}$ with $0 \notin \operatorname{supp}(\mu)$. The following are equivalent,

1. $\gamma(\mathbf{M})=0$.

2. For all $k \in \mathbb{Z}, z^{k} \in \overline{\left[z^{k+1}, z^{k+2}, \ldots\right]} L^{2}(\mu)$.

Proof. First, there exist $R>0$ and $\alpha>0$ such that $\alpha \leq|z| \leq R$ for every $z \in \operatorname{supp}(\mu)$. Consequently, for every $k \in \mathbb{Z}$ and for every $v_{0}, v_{1}, \ldots, v_{n} \in \mathbb{C}, n \in \mathbb{N}$, it follows that

$$
\begin{gathered}
\alpha^{2 k} \int\left|1-v_{0}-v_{1} z-\cdots-v_{n} z^{n}\right|^{2} d \mu \leq \int|z|^{2 k}\left|1-v_{0}-v_{1} z-\cdots-v_{n} z^{n}\right|^{2} d \mu= \\
\int\left|z^{k}-v_{0} z^{k+1}-v_{1} z^{k+1}-\cdots-v_{n} z^{k+n}\right|^{2} d \mu \leq R^{2 k} \int\left|1-v_{0}-v_{1} z-\cdots-v_{n} z^{n}\right|^{2} d \mu .
\end{gathered}
$$

Therefore, $\mathbf{1} \in P_{0}^{2}(\mu)$ if and only if $z^{k} \in \overline{\left[z^{k+1}, z^{k+2}, \ldots\right]}{ }^{L^{2}(\mu)}$, for all $k \in \mathbb{Z}$.

Consequently, for compactly supported measures with $0 \notin \operatorname{supp}(\mu)$, the condition $\gamma(\mathbf{M})=0$ characterizes completeness of polynomials in the closed subspace of Laurent polynomials in $L^{2}(\mu)$ denoted by $\mathbb{C}\left[z, z^{-1}\right]=\overline{\left[1, z, \frac{1}{z}, z^{2}, \frac{1}{z^{2}}, \ldots\right]}{ }^{L^{2}(\mu)}$ :

Corollary 2. Let $\mu$ be a non-trivial positive compactly supported measure in $\mathcal{T}$ with $0 \notin \operatorname{supp}(\mu)$. The following are equivalent,

1. $\gamma(\mathbf{M})=0$.

2. $P^{2}(\mu)=\mathbb{C}\left[z, z^{-1}\right]$.

In particular, for non-trivial positive measures $\sigma$ supported in the unit circle, it is well-known that Laurent polynomials are dense in $L^{2}(\sigma)$ and therefore the condition $\gamma(\mathbf{M})=0$ characterizes completeness of polynomials in $L^{2}(\sigma)$. More generally, Corollary 2 will be true whenever Laurent polynomials are dense in $L^{2}(\mu)$. Moreover, we have:

Theorem 1. Let $\Gamma$ be a Jordan curve such that $0 \in \operatorname{int} \Gamma$ and let $\mu$ be a measure with support in $\Gamma$ and associated moment matrix $\mathbf{M}$. The following are equivalent:

1. $\gamma(\mathbf{M})=0$.

2. $P^{2}(\mu)=L^{2}(\mu)$.

Proof. In [1], the following consequence of Mergelyan's theorem is given: if $\Gamma$ is a Jordan curve, $0 \in \operatorname{int} \Gamma$, and $f$ is continuous on $\Gamma$, then for every $\epsilon>0$ there exists a $P(z)=\sum_{n=-N}^{N} a_{n} z^{n}$ such 
that $|f(z)-P(z)|<\epsilon$ for every $z \in \Gamma$. This means that $\mathbb{C}\left[z, z^{-1}\right]$ is dense in the space of continuous functions on $\Gamma$ with the uniform norm. Therefore,

$$
\int|f(z)-P(z)|^{2} \leq \epsilon^{2} \mu(\Gamma)
$$

and, consequently, $\mathbb{C}\left[z, z^{-1}\right]$ is dense in the space of continuous functions in $L^{2}(\mu)$. Since, for compactly supported measures, continuous functions are dense in $L^{2}(\mu)$, we obtain that $P^{2}(\mu)=\mathbb{C}\left[z, z^{-1}\right]=$ $L^{2}(\mu)$ if and only if $\gamma(\mathbf{M})=0$ as we required.

As a consequence of the above results, we have the well-known consequence of Szegö theorem for measures supported in the unit circle:

Corollary 3. Let $\sigma$ be a non-trivial positive measure with support in $\mathbb{T}$ and $\left\{\varphi_{n}(z)\right\}_{n=0}^{\infty}$ the associated orthonormal polynomial sequence. Then, the following conditions are equivalent

1. Polynomials are dense in $L^{2}(\mathbb{T})$.

2. $\sum_{k=0}^{\infty}\left|\varphi_{k}(0)\right|^{2}=\infty$.

Proof. The result is a consequence of Theorem 1 and Proposition 3 since

$$
\gamma(\mathbf{M})=\frac{1}{\sum_{n=0}^{\infty}\left|\varphi_{n}(0)\right|^{2}},
$$

where $\gamma(\mathbf{M})=0$ whenever $\sum_{n=0}^{\infty}\left|\varphi_{n}(0)\right|^{2}=\infty$.

\section{Bounded Point Evaluations from the Matrix Algebra Point of View: Thomson's Theorem Revisited}

We first recall the definitions of bounded point evaluation. Let $\mu$ be a non-trivial positive measure with support on $\mathbb{C}$. Recall (see, e.g., [11]) that a point $z_{0} \in \mathbb{C}$ is a bounded point evaluation (bpe) for $P^{2}(\mu)$ if there exists a constant $C>0$ such that for every polynomial $p(z)$

$$
\left|p\left(z_{0}\right)\right| \leq C\left(\int|p(z)|^{2} d \mu\right)^{1 / 2} .
$$

Moreover, the point $z_{0} \in \mathbb{C}$ is an analytic bounded point evaluation (abpe) if there exists a constant $C>0$ and $\epsilon>0$ such that for every $w \in \mathbb{C}$ with $\left|w-z_{0}\right|<\epsilon$ and for every polynomial $p(z)$ it holds

$$
|p(w)|^{2} \leq C \int|p(z)|^{2} d \mu
$$

Remark 4. Of course, an analytic bounded point evaluation is a bounded point evaluation. The converse is not true; indeed, any atomic isolated point is a bounded point evaluation but it is not an analytic bounded point evaluation.

It is well-known that, if a point $z_{0} \in \operatorname{supp}(\mu)$ is an atomic point of $\mu$, that is $\mu\left(\left\{z_{0}\right\}\right)>0$, then it is a bounded point evaluation for $P^{2}(\mu)$. We prove it for the sake of completeness

Lemma 4. Let $z_{0}$ be an atomic point of a measure $\mu$ with $\mu\left(\left\{z_{0}\right\}\right)=\alpha>0$. Then, $z_{0}$ is a bounded point evaluation for $P^{2}(\mu)$ with constant $C=\alpha^{-1 / 2}$.

Proof. Let $p(z)$ be a polynomial. Then,

$$
\left|p\left(z_{0}\right)\right|^{2} \mu\left(\left\{z_{0}\right\}\right)=\int_{\left\{z_{0}\right\}}|p(z)|^{2} d \mu \leq \int|p(z)|^{2} d \mu .
$$

Therefore, 


$$
\left|p\left(z_{0}\right)\right| \leq \frac{1}{\alpha^{1 / 2}}\left(\int|p(z)|^{2} d \mu\right)^{1 / 2}
$$

Our aim in this section is to prove a theorem that is close in spirit to Thomson's theorem $[7,8]$, but for measures supported in Jordan curves, with the novelty of using techniques from the matrix algebra and using infinite HPD matrices. To do it, we first give a new approach of bounded point evaluations for a measure, and, more generally, for infinite HPD matrices.

Definition 3. Let $\mathbf{M}$ be an HPD matrix and let $P^{2}(\mathbf{M})$ be the closure of the polynomials with the inner product induced by $\mathbf{M}$. Let $z_{0} \in \mathbb{C}$; we say that $z_{0}$ is a bounded point evaluation for $P^{2}(\mathbf{M})$ if there exists a constant $C>0$ such that for every polynomial $p(z)$ it holds that

$$
\left|p\left(z_{0}\right)\right| \leq C\|p(z)\|_{P^{2}(\mathbf{M})}
$$

Remark 5. Obviously, in the case of $\mathbf{M}$ being a moment matrix associated with a measure $\mu$, the notion of bounded point evaluation for $P^{2}(\mathbf{M})$ coincides with the usual of bounded point evaluation for $P^{2}(\mu)$.

We need to introduce a new index for a given $z_{0} \in \mathbb{C}$ :

Definition 4. Let $\mathbf{M}$ be an HPD matrix, $z_{0} \in \mathbb{C}$, and $k_{z_{0}}=\left\{z_{0}^{k}\right\}_{k=0}^{\infty}$. We define

$$
\gamma_{z_{0}}(\mathbf{M})=\inf \left\{v \mathbf{M} v^{*}: \sum_{k=0}^{\infty} v_{k} z_{0}^{k}=1, \quad v \in c_{00}\right\} \text {. }
$$

Remark 6. Note that $\gamma_{z_{0}}(\mathbf{M}) \geq 0$ for every $z_{0} \in \mathbb{C}$ and in the particular case that $z_{0}=0$, then $\gamma_{z_{0}}(\mathbf{M})=\gamma(\mathbf{M})$.

Next we prove:

Lemma 5. Let $\mathbf{M}$ be an HPD matrix. Then, the following statements are equivalent:

1. $z_{0}$ is a bounded point evaluation for $P^{2}(\mathbf{M})$.

2. $\gamma_{z_{0}}(\mathbf{M})>0$.

Proof. Assume first that $z_{0}$ is a bounded point evaluation of $P^{2}(\mathbf{M})$ with constant $C$; then, for every $\left(v_{0}, v_{1}, \ldots, v_{n}, 0,0, \ldots\right)$ and $p(z)=v_{0}+v_{1} z+\ldots v_{n} z^{n}$,

$$
\left|v k_{z_{0}}\right|=\left|v_{0}+v_{1} z_{0}+\cdots+v_{n} z_{0}^{n}\right| \leq C\|p(z)\|_{P^{2}(\mathbf{M})}=C\|v\|_{\mathbf{M}}
$$

In particular, if $v k_{z_{0}}=1$, it holds that $\|v\|_{\mathbf{M}} \geq \frac{1}{C}$ and consequently $\gamma_{z_{0}}(\mathbf{M}) \geq \frac{1}{C}>0$. On the other hand, if $\gamma_{z_{0}}(\mathbf{M})>0$ and $p(z)=\sum_{k=0}^{n} v_{k} z^{k}$ with $\left(v_{0}, \ldots, v_{n}, 0, \ldots\right) \in c_{00}$, either $p\left(z_{0}\right)=0$ and obviously $p\left(z_{0}\right) \leq\|p(z)\|_{P^{2}(\mathbf{M})}$, or $p\left(z_{0}\right) \neq 0$ and the vector $w=\left(w_{i}\right)_{i=0} \in c_{00}$ defined by $w_{i}=\frac{v_{i}}{p\left(z_{0}\right)}$ for each $i \geq 0$ verifies $w k_{z_{0}}=1$ and consequently

$$
\gamma_{z_{0}}(\mathbf{M}) \leq w \mathbf{M} w^{*}=\frac{1}{\left|p\left(z_{0}\right)\right|^{2}} v \mathbf{M} v^{*}=\frac{1}{\left|p\left(z_{0}\right)\right|^{2}}\|p(z)\|_{\mathbf{M}}^{2}
$$

Therefore,

$$
\left|p\left(z_{0}\right)\right|^{2} \leq \frac{1}{\gamma_{z_{0}}(\mathbf{M})}\|p(z)\|_{P^{2}(\mathbf{M})}^{2}
$$


and $z_{0}$ is a bounded point evaluation for $P^{2}(\mathbf{M})$ with constant $\frac{1}{\left(\gamma_{z_{0}}(\mathbf{M})\right)^{1 / 2}}$.

Remark 7. Note that proof of the above Lemma gives us information about the constant of the bounded point evaluation; indeed, $z_{0}$ is bounded point evaluation for $P^{2}(\mathbf{M})$ with constant $\gamma_{z_{0}}(\mathbf{M})$.

We may generalize the notion of kernels in the context of infinite HPD matrices. More precisely, for an infinite HPD matrix $\mathbf{M}$, we may define the associated kernels:

$$
K_{\mathbf{M}}(z, w)=\sum_{n=0}^{\infty} \varphi_{n}(z) \overline{\varphi_{n}(w)},
$$

for every $z, w$ such that the series converges. In this context, the extremal property for polynomials can be reformulated as:

Lemma 6. Let $\mathbf{M}$ be an HPD matrix and let $\left\{\varphi_{n}(z)\right\}_{n=0}^{\infty}$ the sequence of orthonormal polynomials associated with $\mathbf{M}$. For every $z_{0} \in \mathbb{C}$,

$$
\gamma_{z_{0}}(\mathbf{M})=\frac{1}{\sum_{k=0}^{\infty}\left|\varphi_{k}\left(z_{0}\right)\right|^{2}}
$$

Proof. Using the notation for polynomials, we may rewrite:

$$
\gamma_{z_{0}}(\mathbf{M})=\inf \left\{\|p(z)\|_{P^{2}(\mathbf{M})}^{2}: p(z) \in \mathbb{P}[z], p\left(z_{0}\right)=1\right\} .
$$

First, consider a polynomial $q(z)=\sum_{k=0}^{n} v_{k} z^{k}$ and we express it in terms of the orthonormal basis, that is, $q(z)=\sum_{k=0}^{n} w_{k} \varphi_{k}(z)$. Then, by using the Cauchy-Schwartz inequality,

$$
1=\left|q\left(z_{0}\right)\right| \leq\left(\sum_{k=0}^{n}\left|w_{k}\right|^{2}\right)^{1 / 2}\left(\sum_{k=0}^{n}\left|\varphi_{k}\left(z_{0}\right)\right|^{2}\right)^{1 / 2} .
$$

Then,

$$
\frac{1}{\sum_{k=0}^{n}\left|\varphi_{k}\left(z_{0}\right)\right|^{2}} \leq \sum_{k=0}^{n}\left|w_{k}\right|^{2}=\|q(z)\|_{P^{2}(\mathbf{M})}^{2} .
$$

By taking the infimum all over the polynomials of degree $n$,

$$
\frac{1}{\sum_{k=0}^{n}\left|\varphi_{k}\left(z_{0}\right)\right|^{2}} \leq \inf \left\{\|q(z)\|^{2}, q(z) \in \mathbb{P}_{n}[z], q\left(z_{0}\right)=1\right\}
$$

On the other hand, if we consider the polynomial $q(z)=K_{\mathbf{M}}\left(z, z_{0}\right)=\sum_{k=0}^{n} \varphi_{k}(z) \overline{\varphi_{k}\left(z_{0}\right)}$, the above infimum is reached at this polynomial since

$$
\|q(z)\|_{P^{2}(\mathbf{M})}^{2}=\frac{1}{\sum_{k=0}^{n}\left|\varphi_{k}\left(z_{0}\right)\right|^{2}}
$$

Then, for every $n \in \mathbb{N}$,

$$
\begin{aligned}
\gamma_{z_{0}}(\mathbf{M}) & =\inf _{n} \min \left\{\|q(z)\|^{2}, q(z) \in \mathbb{P}_{n}[z], q\left(z_{0}\right)=1\right\} \\
& =\inf _{n} \frac{1}{\sum_{k=0}^{n}\left|\varphi_{k}\left(z_{0}\right)\right|^{2}}=\frac{1}{\sum_{k=0}^{\infty}\left|\varphi_{k}\left(z_{0}\right)\right|^{2}}
\end{aligned}
$$

We summarize all the equivalent notions of bounded point evaluations for an HPD matrix in the following proposition: 
Corollary 4. Let $\mathbf{M}$ be an HPD matrix and let $\left\{\varphi_{n}(z)\right\}_{n=0}^{\infty}$ be the orthonormal polynomial sequence associated with $\mathbf{M}$. Then, the following are equivalent:

1. $z_{0}$ is a bounded point evaluation of $P^{2}(\mathbf{M})$.

2. $K_{\mathbf{M}}\left(z_{0}, z_{0}\right)=\sum_{n=0}^{\infty}\left|\varphi_{n}\left(z_{0}\right)\right|^{2}<\infty$

3. $\gamma_{z_{0}}(\mathbf{M})>0$.

4. $\frac{1}{\gamma_{z_{0}}(\mathbf{M})}=\lim _{n \rightarrow \infty}\left(1, z_{0}, z_{0}^{2}, \ldots, z_{0}^{n}\right) \mathbf{M}_{n}^{-1}\left(\begin{array}{c}1 \\ z_{0} \\ \vdots \\ z_{0}^{n}\end{array}\right)>0$.

Proof. We only need to prove $(2) \Longleftrightarrow(4)$. In [2], the expression of the $n$-kernel by determinants is given as follows

$$
K_{n}(y, z)=-\frac{1}{\Delta_{n}}\left|\begin{array}{ccccc}
c_{00} & c_{10} & \ldots & c_{n, 0} & 1 \\
c_{01} & c_{11} & \ldots & c_{n, 1} & \bar{z} \\
\vdots & \vdots & \ddots & \vdots & \vdots \\
c_{0, n} & c_{1, n} & \ldots & c_{n, n} & \bar{z}^{n} \\
1 & y & \ldots & y^{n} & 0
\end{array}\right|=\left(1, y, \ldots, y^{n}\right) \mathbf{M}_{n}^{-1}\left(\begin{array}{c}
1 \\
\bar{z} \\
\vdots \\
\bar{z}^{n}
\end{array}\right)
$$

the last identity is the Schur complement, which says $\left|\begin{array}{cc}A & b \\ c^{t} & 0\end{array}\right|=|A|\left\langle c, A^{-1} b\right\rangle$.

As a consequence of this result, we obtain our main result, which is the following proof of Thomson's theorem for measures supported in Jordan curves via an algebraic way. This allows us to provide an algebraic characterization of density of polynomials in terms of an index of the moment matrix associated with the measure:

Theorem 2. Let $\Gamma$ be a Jordan curve such that $z_{0} \in \operatorname{int} \Gamma$ and let $\mu$ be a measure with infinite support in $\Gamma$ with associated moment matrix $\mathbf{M}$. Then, the following statements are equivalent

1. $\gamma_{z_{0}}(\mathbf{M})>0$.

2. $P^{2}(\mu) \neq L^{2}(\mu)$.

3. $z_{0}$ is a bounded point evaluation of $P^{2}(\mathbf{M})$.

Proof. Let $\widetilde{\mathbf{M}}$ be the moment matrix associated with image measure $\tilde{\mu}$ obtained after a similarity map, $\varphi(z)=\alpha z+\beta$ onto $\mathbb{C}$, is applied to the measure $\mu$ and $\tilde{\Gamma}$ the image Jordan curve.

We first prove that

$$
\gamma(\widetilde{\mathbf{M}})=\gamma_{z_{0}}(\mathbf{M}) .
$$

An expression that relates the matrices $\mathbf{M}_{n}$ and $\widetilde{\mathbf{M}}_{n}$ is known, which is given by (see, e.g., [12])

$$
\widetilde{M}_{n}=\mathbf{A}_{n}^{*}(\alpha, \beta) \mathbf{M}_{n} \mathbf{A}_{n}(\alpha, \beta),
$$

where $\mathbf{A}_{n}(\alpha, \beta)$ is defined as in [12],

$$
\mathbf{A}_{n}(\alpha, \beta)=\left(\begin{array}{ccccc}
\left(\begin{array}{c}
0 \\
0
\end{array}\right) \alpha^{0} \beta^{0} & \left(\begin{array}{c}
1 \\
0
\end{array}\right) \alpha^{0} \beta^{1} & \left(\begin{array}{c}
2 \\
0
\end{array}\right) \alpha^{0} \beta^{2} & \left(\begin{array}{c}
3 \\
0
\end{array}\right) \alpha^{0} \beta^{3} & \ldots \\
0 & \left(\begin{array}{l}
1 \\
1
\end{array}\right) \alpha^{1} \beta^{0} & \left(\begin{array}{l}
2 \\
1
\end{array}\right) \alpha^{1} \beta^{1} & \left(\begin{array}{c}
3 \\
1 \\
1
\end{array}\right) \alpha^{1} \beta^{2} & \ldots \\
0 & 0 & \left(\begin{array}{c}
2 \\
2
\end{array}\right) \alpha^{2} \beta^{0} & \left(\begin{array}{c}
3 \\
2
\end{array}\right) \alpha^{2} \beta^{1} & \ldots \\
0 & 0 & 0 & \left(\begin{array}{c}
3 \\
3
\end{array}\right) \alpha^{3} \beta^{0} & \ldots \\
\vdots & \vdots & \vdots & \vdots & \ddots
\end{array}\right) .
$$


Note that, if we choose $\alpha=1$ and a translation $\beta=-z_{0}$, then $0 \in \operatorname{int}(\tilde{\Gamma})$, and we obtain:

$$
\widetilde{\mathbf{M}_{n}}=\mathbf{A}_{n}^{*}\left(1,-z_{0}\right) \mathbf{M}_{n} \mathbf{A}_{n}\left(1,-z_{0}\right) .
$$

Since $\mathbf{M}$ is HPD, all its sections are invertible, and we can write

$$
\widetilde{\mathbf{M}}_{n}^{-1}=\mathbf{A}_{n}^{-1}\left(1,-z_{0}\right) \mathbf{M}_{n}^{-1}\left[\mathbf{A}_{n}^{*}\right]^{-1}\left(1,-z_{0}\right) .
$$

It is clear that

$$
\begin{aligned}
\mathbf{A}_{n}\left(1,-z_{0}\right)= & \left(\begin{array}{cccccc}
1 & -z_{0} & z_{0}^{2} & -z_{0}^{3} & \ldots & \pm\left(\begin{array}{c}
n \\
n
\end{array}\right) z_{0}^{n} \\
0 & 1 & -2 z_{0} & 3 z_{0}^{2} & \ldots & \mp\left(\begin{array}{c}
n \\
n-1
\end{array}\right) z_{0}^{n-1} \\
0 & 0 & 1 & -3 z_{0} & \ldots & \pm\left(\begin{array}{c}
n \\
n-2
\end{array}\right) z_{0}^{-1} \\
\vdots & \vdots & \vdots & \vdots & & \vdots \\
0 & 0 & 0 & 0 & \ldots & \left(\begin{array}{c}
n \\
0
\end{array}\right) z^{0}
\end{array}\right) \Rightarrow \\
\mathbf{A}_{n}^{-1}\left(1,-z_{0}\right) & =\left(\begin{array}{cccccc}
1 & z_{0} & z_{0}^{2} & z_{0}^{3} & \ldots & \left(\begin{array}{c}
n \\
n \\
n
\end{array}\right) z_{0}^{n} \\
0 & 1 & 2 z_{0} & 3 z_{0}^{2} & \ldots & \left(\begin{array}{c}
n \\
n-1 \\
n
\end{array}\right) z_{0}^{n-1} \\
0 & 0 & 1 & 3 z_{0} & \ldots & \left(\begin{array}{c}
n \\
n-2
\end{array}\right) z_{0}^{-1} \\
\vdots & \vdots & \vdots & \vdots & & \vdots \\
0 & 0 & 0 & 0 & \ldots & \left(\begin{array}{c}
n \\
0
\end{array}\right) z^{0}
\end{array}\right)
\end{aligned}
$$

It is immediate that

$$
\gamma(\widetilde{\mathbf{M}})=\lim _{n} \frac{1}{e_{0}^{t} \widetilde{\mathbf{M}}_{n}^{-1} e_{0}}
$$

and

$$
e_{0}^{t} \widetilde{\mathbf{M}}_{n}^{-1} e_{0}=e_{0}^{t}\left(\mathbf{A}_{n}^{-1}\left(1,-z_{0}\right) \mathbf{M}_{n}^{-1}\left[\mathbf{A}_{n}^{*}\right]^{-1}\left(1,-z_{0}\right)\right) e_{0} .
$$

We have also that

$$
e_{0}^{t} \mathbf{A}_{n}^{-1}\left(1,-z_{0}\right)=\left(1, z_{0}, z_{0}^{2}, \ldots, z_{0}^{n}\right), \quad\left[\mathbf{A}_{n}^{*}\right]^{-1}\left(1,-z_{0}\right) e_{0}=\left(1, \bar{z}_{0}, \bar{z}_{0}^{2}, \ldots, \bar{z}_{0}^{n}\right)^{t} .
$$

Consequently,

$$
\gamma(\widetilde{\mathbf{M}})=\gamma_{z_{0}}(\mathbf{M}) .
$$

Now, the result is consequence of Theorem 1.

We finish with some applications to our results:

Corollary 5. Let $\Gamma$ be an analytic Jordan curve with non-empty interior and let $\mu$ be a measure with support in $\Gamma$. Let $d \mu=\rho(z)|d z|$, where $\rho(z)$ is positive and continuous function on $\Gamma$. Then, $L^{2}(\mu) \neq P^{2}(\mu)$.

Proof. Let $z_{0}$ be an arbitrary interior point of $\Gamma$; by using the results in [2], it follows that $K_{\mathbf{M}}\left(z_{0}, z_{0}\right)<$ $\infty$. Therefore, $\gamma_{z_{0}}(\mathbf{M})>0$, and ,by using Theorem 1 , we may conclude $L^{2}(\mu) \neq P^{2}(\mu)$.

In addition, from Theorem 3, the following result is obvious:

Corollary 6. Let $\Gamma$ be a Jordan curve and $\mu$ be a measure with support in $\Gamma$. Assume that $L^{2}(\mu) \neq P^{2}(\mu)$, then every $z_{0} \in$ int $\Gamma$ is a bounded point evaluation of $\mu$. 
Author Contributions: All authors contributed equally to this work, as well as to its preparation. They have read and approved the final manuscript.

Funding: This research was funded by Ministerio de Economía, Industria and Competitividad of Spain, Grant MTM2016-80582-R (AEI/FEDER, UE).

Conflicts of Interest: The authors declare no conflict of interest.

\section{References}

1. Gaier, D. Lectures on Complex Approximation; Birkhauser: Boston, MA, USA, 1987.

2. Szegö, G. Orthogonal Polynomials; American Mathematical Society, Colloquium Publications: Providence, RI, USA, 1975; Volume 23.

3. Simon, B. Orthogonal Polynomials on the Unit Circle, Part 1. Classical Theory; 54 Part 1; American Mathematical Society, Colloquium Publications: Providence, RI, USA, 2003.

4. Escribano, C.; Gonzalo, R.; Torrano, E. Small Eigenvalues of Large Hermitian moment matrices. J. Math. Anal. Appl. 2011, 374, 470-480. [CrossRef]

5. Berg, C.; Duran, A.J. Orthogonal Polynomials and analytic functions associated with positive definite matrices. J. Math. Anal. Appl. 2006, 315, 54-67. [CrossRef]

6. Daruis, L.; Hernández, J.; Marcellán, F. Spectral transformations for Hermitian Toeplitz matrices. J. Comput. Appl. Math. 2007, 202, 155-176. [CrossRef]

7. Brennan, J.E. Thomson's Theorem on mean square polynomial approximation. St. Petesburg Math. J. 2006, 17, 217-238. [CrossRef]

8. Thomson, J.E. Appoximation in the mean by polynomials. Ann. Mater. 1991, 133, 477-507. [CrossRef]

9. Grenander, U.; Szegö, G. Toeplitz Forms and Their Applications; Chelsea Publishing Company: New York, NY, USA, 1958.

10. Nikishin, E.M.; Sorokin, V.N. Rational Approximations and Orthogonality; Translations of Mathematical Monographs; American Mathematical Society: Providence, RI, USA, 1991; Volume 92.

11. Conway, J.B. The Theory of Subnormal Operators; Mathematical Surveys and Monographs; American Mathematical Society: Providence, RI, USA, 1985; Volume 36.

12. Escribano, C.; Sastre, M.A.; Torrano, E. A fixed point theorem for moment matrices self-similar measures. J. Comput. Appl. Math. 2007, 207, 352-359. [CrossRef]

(C) 2019 by the authors. Licensee MDPI, Basel, Switzerland. This article is an open access article distributed under the terms and conditions of the Creative Commons Attribution (CC BY) license (http:/ / creativecommons.org/licenses/by/4.0/). 